\title{
EFEITO ANTIOXIDANTE DA FICOCIANINA EM PESCADO SALGADO-SECO
}

\author{
Antioxidant effect of phycocianin on dried-salted fish \\ Telma Elita Bertolin ${ }^{1}$, Cíntia Guarienti², Daniele Farias², Fernanda Taís Souza², \\ Luiz Carlos Gutkoski ${ }^{2}$, Luciane Maria Colla ${ }^{2}$
}

\begin{abstract}
RESUMO
A conservação e o armazenamento de alimentos com elevados teores lipídicos podem conduzir ao desencadeamento do processo oxidativo, causando modificações nas características sensoriais e redução da vida útil dos mesmos. A ficocianina é um pigmento extraído da microalga Spirulina platensis que apresenta propriedades antioxidantes, por isso, objetivou-se verificar o efeito antioxidante da ficocianina durante o armazenamento do pescado salgado. O pescado salgado foi produzido com o peixe Pacu (Piaractus mesopotamicus, Holmberg, 1887), o qual foi submetido a 5 tratamentos ( $\mathrm{T}_{1}$ : sem adição de antioxidante, $\mathrm{T}_{2}$ : com adição de antioxidante sintético BHT na concentração de $0,01 \%, \mathrm{~T}_{3} \mathrm{~T}_{4}$ e $\mathrm{T}_{5}$ : com a adição de ficocianina nas concentrações de $0,5 \%, 1 \% \mathrm{e}$ $1,5 \%$, respectivamente). As amostras foram analisadas nos tempos: zero, $30 \mathrm{~d}$ e $60 \mathrm{~d}$ de armazenamento através do índice de TBA. Pelos resultados observou-se que a presença do antioxidante natural ficocianina ocasionou uma inibição no processo de oxidação lipídica, com índices de TBA, estatisticamente iguais $(\mathrm{p}>0,05)$ ao tratamento com uso de antioxidante sintético.
\end{abstract}

Termos de indexação: Peroxidação lipídica, processo oxidativo.

\section{ABSTRACT}

Conservation and storage of food with a lipidic content may unchain oxidative processes, causing modifications of sensorial characteristics and reduction of useful life. Phycocianin is a pigment of Spirulina platensis, which presents antioxidant proprieties. The objective of this work was to verify the antioxidant effect of phycocyanin during the storage of salted fish. The salted fish was produced with the pacu species (Piaractus mesopotamicus Holmberg, 1887), which was submitted to 5 treatments (T1: without addition of antioxidant; T2: with addition of synthetic antioxidant BHT in the concentration of $0.01 \%, \mathrm{~T} 3$, T4 and T5: with addition of phycocianin at concentrations of $0.5 \%, 1 \%$ and $1.5 \%$ respectively). Samples were analyzed at zero (0), 30 and 60 days of storage determining the physics-chemical quality through the index of TBA. The results showed that the addition of the natural antioxidant phycocianin caused the inhibition of the process of oxidation, with TBA index statistically equal ( $p>0.05)$ of that obtained with the use of synthetic antioxidant.

Index terms: Lipidic peroxidation, oxidative process.

(Recebido em 11 de setembro de 2008 e aprovado em 29 de outubro de 2009)

\section{INTRODUÇÃO}

A oxidação de lipídios, ou autoxidação, inicia-se com a formação de radicais livres, formando hidroperóxidos, os quais podem causar alterações sensoriais indesejáveis em óleos, gorduras ou alimentos, produzindo odor e sabor desagradáveis e com isso, a diminuição do tempo de vida útil (WICK et al., 2001). Além disso, os produtos da oxidação lipídica podem desencadear a peroxidação in vivo, a diferentes classes de moléculas biológicas, resultando em situações que podem variar desde o envelhecimento precoce até a instalação de patologias, como doenças degenerativas, dentre outras (FINKEL; HOLBROOK, 2000).

A carne de pescado apresenta-se como uma fonte importante de proteínas, vitaminas, minerais e lipídios essenciais para a alimentação humana. Esse alimento, no entanto, é mais perecível que a carne de frango ou carne vermelha em função de seus teores de aminoácidos livres, bases nitrogenadas e ácidos graxos insaturados (ASHIE et al., 1996; GONZÁLEZ-FANDOS et al., 1994). Os ácidos graxos polinsaturados encontrados em lipídios de pescado torna-os suscetíveis à oxidação, levando à formação de peróxidos e produtos da degradação desses. As insaturações representam o centro ativo pelo qual se inicia a reação de oxidação. Do ponto de vista da deterioração do sabor dos alimentos, os peróxidos não são importantes, e sim, os produtos oriundos de sua decomposição (aldeídos, cetonas, álcoois, hidrocarbonetos e ácidos), os quais podem ser detectados através do índice de TBA (ARAÚJO, 2004).

${ }^{1}$ Instituto de Ciências Biológicas/ICB - Universidade de Passo Fundo/UPF - BR 285 - São José - Cx. P. 611 - $99052-900$ - Passo Fundo, RS telma@upf.br

${ }^{2}$ Faculdade de Engenharia e Arquitetura/FEAR - Universidade de Passo Fundo/UPF - Passo Fundo, RS 
Segundo Hasenhuettl e Wan (1992), citado por Petenuci et al. (2010) estes produtos oriundos da degradação dos peróxidos são responsáveis pelas características organolépticas e físico-químicas associadas com a rancificação e, desta forma, afetam a qualidade do produto, tornando-o impróprio para consumo.

O índice de TBA (ácido 2-tiobarbitúrico) é utilizado no controle de qualidade de óleos, gorduras, e de produtos ricos em óleos e gorduras, por fornecer informações essenciais sobre o estado oxidativo e na predição da rancidez do alimento analisado. Essa análise quantifica o malonaldeído (MDA), um dos produtos da decomposição dos hidroperóxidos de ácidos graxos poliinsaturados, formados durante o processo oxidativo. O MDA (malonaldeído) é um dialdeído de três carbonos, com grupos carbonilas nos carbonos C-1 e C-3. A reação envolve o ácido 2-tiobarbitúrico com o malonaldeído produzindo um composto de cor vermelha, medido espectrofotometricamente em comprimentos de onda de $532 \mathrm{~nm}$ (OSAWA et al., 2005).

A inibição ou atenuação da peroxidação dos lipídios e também a oxidação de outras moléculas, como proteínas e DNA, pode ser realizada pelo uso de substâncias com poder antioxidante. Os antioxidantes atuam prevenindo a deterioração oxidativa de alimentos (ADEGOKE et al., 1998) e podem ser naturais ou sintéticos. Na atualidade, percebese um crescente interesse da indústria de alimentos na aplicação de substâncias antioxidantes naturais em substituição aos antioxidantes sintéticos, com apelo à saúde humana (DURAN; PADILHA, 1993; RAMALHO; JORGE, 2006). A Spirulina platensis é uma microalga verdeazulada filamentosa fotoautotrófica. Seus filamentos multicelulares podem ter de 50-300 $\mu \mathrm{m}$ de comprimento e $10 \mu \mathrm{m}$ de diâmetro. Sua forma em espiral originou seu nome (RICHMOND, 1990). Atualmente, a microalga Spirulina platensis é utilizada principalmente como suplemento alimentar, devido aos benefícios de seu consumo, indicando um grande potencial na alimentação humana e ração animal (HENRIKSON, 1994). Segundo Alencar et al. (2011), entre todos os vegetais, a cianofícea Spirulina é a mais rica fonte de ferro e proteína, contendo oito dos dez aminoácidos essenciais, usados para combater, respectivamente, a anemia e a má nutrição, prevalentes na população mundial. A presença de outros minerais importantes para o metabolismo humano torna a Spirulina um alimento completo por reunir um grande número de propriedades benéficas, dificilmente encontradas em um único produto natural. Essa microalga é classificada como GRAS (Generally Recognized as Safe) pelo FDA (Food and Drug Administration), o que garante seu uso como alimento sem riscos à saúde. A ficocianina e a aloficocianina são pigmentos encontrados nos tilacóides de cianobactérias, como a Spirulina platensis, e são estudados devido à capacidade de reagir com as substâncias reativas ao oxigênio geradas durante o processo oxidativo (ESTRADA et al., 2001). A ficocianina é o principal pigmento da microalga, podendo chegar a $20 \%$ em peso seco (HENRIKSON, 1994). Diversos fatores podem influenciar a extração de ficocianina. Os mais importantes são o método de ruptura celular, o tipo de solvente, a proporção de biomassa e solvente e também o tempo de extração (ABALDE et al., 1995) A ficocianina, além de apresentar propriedades antioxidantes (MOREIRA, 2004; SOUZA et al., 2006; PILATTI et al., 2007; CANFIELD et al., 2008), é utilizada na indústria de alimentos principalmente na coloração de doces, sorvetes, produtos lácteos e bebidas não alcoólicas (RICHMOND, 1990).

Objetivou-se verificar o potencial antioxidante da ficocianina, durante o armazenamento do pescado salgadoseco.

\section{MATERIAL E MÉTODOS}

O pescado salgado foi elaborado com o peixe de água doce, Pacu (Piaractus mesopotamicus, HOLMBERG,1887). O processo de obtenção do pescado consistiu no abate, na evisceração e na retirada da cabeça dos animais, com posterior processo de salga mista, por um período de $7 \mathrm{~d}$. A quantidade de antioxidante foi baseada no teor de lipídios da matéria-prima, o qual foi determinado de acordo com o método 032/IV do Instituto Adolfo Lutz (1985). A incorporação dos antioxidantes ao pescado foi realizada juntamente com o sal durante o processo de salga. Após a salga foi realizada a secagem do pescado em estufa com circulação de ar a uma temperatura de $36^{\circ} \mathrm{C}$ por um período de $48 \mathrm{~h}$, para atender ao Regulamento Técnico de Identidade e Qualidade de Peixe Salgado (BRASIL, 2000). $\mathrm{O}$ pescado foi armazenado durante um período de $60 \mathrm{~d}$ à temperatura de $25^{\circ} \mathrm{C}$, em ambiente protegido de sujidades. Os tratamentos experimentais foram em número de cinco (5): $\left(\mathrm{T}_{1}\right)$ pescado salgado sem adição de antioxidantes (controle); $\left(\mathrm{T}_{2}\right)$ pescado salgado com adição de $0,01 \%$ do antioxidante sintético butil hidroxitolueno (BHT); $\left(\mathrm{T}_{3}\right)$ pescado salgado com adição de ficocianina na concentração de $0,5 \%$; $\left(\mathrm{T}_{4}\right)$ pescado salgado com adição de ficocianina na concentração de $1,0 \%$; $\left(\mathrm{T}_{5}\right)$ pescado salgado com adição de ficocianina na concentração de 1,5\%.

A oxidação lipídica do pescado salgado foi analisada através da determinação do índice de TBA (ácido tiobarbitúrico) segundo Araújo (2004), no tempo zero (retirada da estufa), $30 \mathrm{~d}$ e $60 \mathrm{~d}$ de armazenamento, sendo 
as amostras retiradas de forma aleatória na peça de pescado e analisadas em triplicata.

Os resultados foram avaliados por análise de variância ANOVA e teste de Tukey, a 5\% de significância, utilizando-se o programa Statistica Versão 6.0 (STATSOFT STATISTICA FOR WINDOWS, 2001).

\section{RESULTADOS E DISCUSSÃO}

A Tabela 1 apresenta os resultados do índice de TBA do pescado salgado submetidos aos tratamentos $\mathrm{T}_{1}$ a $\mathrm{T}_{5}$, expresso em $\mathrm{mg} \mathrm{kg}^{-1}$. Com a análise de variância desses dados apresentaram-se diferenças significativas entre as amostras de pescado salgado-seco submetidas aos diferentes tratamentos $(\mathrm{p}<0,001)$.

A comparação de médias dos índices de TBA através do teste de Tukey, no início do período de estocagem (tempo zero) indicou diferenças significativas entre os tratamentos $(\mathrm{p}<0,05)$, sendo que o maior valor encontrado foi para a amostra controle $\left(\mathrm{T}_{1}\right)$ com 15,16 \pm 0,36 ( $\left.\mathrm{mg} \mathrm{kg}^{-1}\right)$, seguido pelos tratamentos $\mathrm{T}_{4}, \mathrm{~T}_{3}, \mathrm{~T}_{5}$ e $\mathrm{T}_{2}$.

Aos $30 \mathrm{~d}$ de armazenamento, verifica-se que os tratamentos com ficocianina $\left(\mathrm{T}_{3}, \mathrm{~T}_{4} \mathrm{e} \mathrm{T}_{5}\right)$ não diferiram entre si a $5 \%$ de significância, no entanto, foram significativamente inferiores ao tratamento controle $\left(\mathrm{T}_{1}\right)$. Os tratamentos $\mathrm{T}_{2}$ (BHT) e $\mathrm{T}_{5}(1,5 \%$ de ficocianina) apresentaram-se estatisticamente iguais no intervalo de $95 \%$ de confiança.

No tempo 60 dias de armazenamento, os tratamentos com $0,5 \%$ e $1 \%$ de ficocianina $\left(\mathrm{T}_{3}\right.$ e $\left.\mathrm{T}_{4}\right)$ com 8,12 e 8,04 $\left(\mathrm{mg} \mathrm{kg}^{-1}\right)$, apresentaram resultados significativamente superiores quando comparados ao resultado obtido com o antioxidante BHT $\left(\mathrm{T}_{2}\right)$ com $6,63\left(\mathrm{mg} \mathrm{kg}^{-1}\right)$, entretanto inferiores ao tratamento controle $\left(\mathrm{T}_{1}\right)$ com $18,35\left(\mathrm{mg} \mathrm{kg}^{-1}\right)$. $\mathrm{O}$ tratamento com $1,5 \%$ de ficocianina $\left(\mathrm{T}_{5}\right)$ apresentou índice de TBA estatisticamente igual $(\mathrm{p}>0,05)$ ao obtido com BHT $\left(\mathrm{T}_{2}\right)$, com valores de 7,36 e 6,63 $\left(\mathrm{mg} \mathrm{kg}^{-1}\right)$, respectivamente. Os tratamentos, $\mathrm{T}_{2}, \mathrm{~T}_{3}, \mathrm{~T}_{4}$ e $\mathrm{T}_{5}$ com antioxidante apresentaram resultados de TBA inferiores ao tratamento controle, $\mathrm{T}_{1}(\mathrm{p}<0,05)$.

Os valores dos índices de TBA verificados no tempo zero de estocagem podem estar indicando que as reações de oxidação lipídica foram desencadeadas na etapa de secagem. Porém, nesse tempo os pescados salgados adicionados de $0,01 \%$ de BHT $\left(\mathrm{T}_{2}\right)$ e $1,5 \%$ de ficocianina $\left(\mathrm{T}_{5}\right)$ apresentaram índices de TBA inferiores aos demais tratamentos. Além disso, pelos resultados observou-se que o tratamento $\mathrm{T}_{5}$ com $1,5 \%$ de ficocianina, foi estatisticamente igual ao $\mathrm{T}_{2}$,com antioxidante sintético padrão (BHT) na inibição da oxidação lipídica.

Resultados semelhantes foram encontrados por Nassu et al. (2003) em estudo da peroxidação lipídica de embutido fermentado com carne de caprino, com óleo de alecrim como antioxidante, nas concentrações de 0,025 e $0,05 \%$. Nos resultados comprovou-se que os valores dos índices de TBA variaram com a mesma tendência deste estudo, ou seja, aumento seguido de diminuição, com valores que variaram de 5 a $15\left(\mathrm{mg} \mathrm{kg}^{-1}\right)$, durante o tempo de maturação que compreendeu 90 dias.

Pereira et al. (2006), em estudo da estabilidade de armazenamento sob temperatura de congelamento da carne de ema (Rhea americana) também verificaram que ocorreu um aumento significativo do número de TBA nas amostras, com variações de 1,5-2,5 mg de TBA kg-1. Apesar da baixa concentração de lipídios existente na carne de ema, parece ocorrer uma susceptibilidade alta à oxidação lipídica; o que pode estar relacionado ao perfil qualitativo e quantitativo dos ácidos graxos existentes nessa carne.

Morcuende et al. (2003), avaliando a estabilidade oxidativa de diferentes músculos da carcaça suína, armazenados sob refrigeração $\left(4^{\circ} \mathrm{C}\right)$ durante $10 \mathrm{~d}$, relatam que os valores de TBA tiveram uma tendência de aumento no período de estocagem dos músculos $(\mathrm{m}$. Masseter 0,39 a $1,13 \mathrm{mg}^{\mathrm{TBA} \mathrm{kg}^{-1}}$; m. Longissimus dorsi - 0,36 a 0,65 mg TBA kg ${ }^{-1} ; \mathrm{m}$. Serratus ventralis - 0,37 a 0,60 mg TBA kg-1).

Tabela 1 - Índice de TBA $\left(\mathrm{mg} \mathrm{kg}^{-1}\right)$ para os tratamentos $\mathrm{T}_{1}$ a $\mathrm{T}_{5}$, durante o tempo de armazenamento do pescado salgado.

\begin{tabular}{cccc}
\hline Tratamentos & \multicolumn{3}{c}{ Tempo $(\mathrm{d})$} \\
\cline { 2 - 4 } & 0 & 30 & 60 \\
\hline Controle $\left(\mathrm{T}_{1}\right)$ & $15,16 \pm 0,36^{\mathrm{d}}$ & $16,54 \pm 0,11^{\mathrm{c}}$ & $18,35 \pm 0,52^{\mathrm{c}}$ \\
$0,01 \%$ BHT $\left(\mathrm{T}_{2}\right)$ & $7,52 \pm 0,17^{\mathrm{a}}$ & $11,52 \pm 0,49^{\mathrm{a}}$ & $6,63 \pm 0,46^{\mathrm{a}}$ \\
$0,5 \%$ ficocianina $\left(\mathrm{T}_{3}\right)$ & $14,18 \pm 0,09^{\mathrm{c}}$ & $13,68 \pm 0,78^{\mathrm{b}}$ & $8,12 \pm 0,13^{\mathrm{b}}$ \\
$1,0 \%$ ficocianina $\left(\mathrm{T}_{4}\right)$ & $15,09 \pm 0,06^{\mathrm{d}}$ & $13,72 \pm 1,20^{\mathrm{b}}$ & $8,04 \pm 0,15^{\mathrm{b}}$ \\
$1,5 \%$ ficocianina $\left(\mathrm{T}_{5}\right)$ & $9,02 \pm 0,15^{\mathrm{b}}$ & $12,09 \pm 0,14 \mathrm{a}^{\mathrm{b}}$ & $7,36 \pm 0,08^{\mathrm{ab}}$ \\
\hline
\end{tabular}

Resultados de média \pm desvio padrão.

Letras diferentes nas colunas representam diferença significativa $(p<0,05)$. 
Badr (2004) avaliando a vida de prateleira da carne de coelho, sob refrigeração $\left(4^{\circ} \mathrm{C}\right)$, relatou que o número de TBA aumentou significativamente durante o período de estocagem $(0,287$ para $0,697 \mathrm{mg}$ de malonaldeído $\mathrm{kg}^{-1}$ amostra).

Sales e Martins (1988) avaliaram o índice de TBA em um período de 45 dias de estocagem em pescado salgadoseco e pescado defumado e encontraram valores variando de 0,06 a 1,94 mg MDA kg-1 de amostra e 0,17 a 2,20 mg MDA kg ${ }^{-1}$ de amostra, respectivamente, demonstrando um aumento do número de TBA com o tempo de estocagem. Estudos realizados por Cozzo et al. (2003), utilizando irradiação e armazenamento refrigerado de filés de Tilápia por um período de 20 dias, apresentaram valores de TBA variando de $0,25 \mathrm{mg}$ de malonaldeído $\mathrm{kg}^{-1}$ de amostra para a amostra não irradiada a $1,36 \mathrm{mg}$ de malonaldeído $\mathrm{kg}^{-1} \mathrm{de}$ amostra para a amostra irradiada com a dose de $5 \mathrm{kGy}$. Beriain et al. (1993) encontraram valores de TBA variando de 1,99 a $36,1\left(\mathrm{mg} \mathrm{kg}^{-1}\right)$ em embutido seco, indicando diferentes níveis de oxidação entre as amostras estudadas. Chen et al. (2007) estudaram a prevenção da oxidação lipídica em filés de peixe e encontraram resultados de inibição da oxidação lipídica depois da suplementação com $\alpha$-tocoferol por 60 dias. Os resultados demonstraram que $900 \mathrm{mg} \mathrm{kg}^{-1}$ de $\alpha$-tocoferol podem prevenir a deterioração lipídica de filés. Sant'Ana e Mancini (2000) estudaram a influência da adição de antioxidantes in vivo na composição de ácidos graxos de filé de Pacu e encontraram os melhores resultados, tanto para a análise de TBA quanto para a análise dos níveis de ácidos graxos individuais, quando usado o tocoferol, superando o antioxidante BHT.

Estes estudos apresentados indicam que a estabilidade lipídica em carnes e produtos cárneos é influenciada por diferentes fatores, dentre eles, a presença de catalisadores da oxidação lipídica, como luminosidade, temperatura, oxigênio, sal, espécie do animal, dieta, estado nutricional e idade do animal antes do abate, tipo de músculo (localização anatômica), manipulação da amostra (processamento, crua, cozida), além do método utilizado para as análises (RHEE et al., 1983).

De acordo com Melton (1990), apesar do malonaldeído ser um produto secundário da oxidação de lipídios, não significa que o número de TBARS continue a aumentar durante a estocagem de produtos cárneos. A diminuição no número de TBARS pode estar relacionada às reações do malonaldeído com as proteínas, durante o período de estocagem.

Com relação à vida de prateleira, de acordo com Kelleher et al. (1994), não há uma quantidade de TBA estabelecida, definindo a ocorrência de oxidação lipídica e/ou indicando que, a partir dela, o pescado não possa ou não deva ser consumido.

Os valores de TBA verificados neste estudo na condição do tratamento controle (sem adição do antioxidante) são altos quando comparados com outros estudos aqui relatados. Porém quando da adição do antioxidante esses valores, no tempo de $60 \mathrm{~d}$ de armazenamento, diminuem significativamente, deixando evidente a atenuação do processo oxidativo. As diferenças nos valores de TBA encontradas nesse trabalho podem estar relacionadas com as condições de armazenamento, tais como, luminosidade, fotoperíodo, temperatura ambiente, em torno de $30^{\circ} \mathrm{C}$ e/ou a presença de sal. $\mathrm{O}$ sal, adicionado durante a manufatura de vários produtos cárneos, tem sido responsabilizado como catalisador da oxidação lipídica, aumentando o número do TBA principalmente se estiver contaminado com metais (HUFFMAN et al., 1981; RHEE et al., 1983; SCHWARTZ; MANDIGO, 1985; Torres et al., 1988, 1989; Asghar et al., 1990).

A peroxidação lipídica manifesta-se de forma mais acentuada no pescado quando há maior conteúdo de lipídios (ITÔ et al., 1969). A composição da gordura que apresenta maior quantidade de ácidos graxos insaturados é mais suscetível à peroxidação lipídica (UNIVERSIDADE FEDERAL DE SÃO PAULO - UNIFESP, 2006). Os altos índices de TBA, encontrados nas amostras deste estudo podem ser decorrentes da porcentagem de lipídeos presentes na amostra (13\%). A Agência Nacional de Vigilância Sanitária (BRASIL, 2000) não estabelece limites de índice de TBA em pescado salgado.

As diversas pesquisas que relatam propriedades antioxidantes de vegetais, de microalgas, entre outros (CANFIELD et al., 2008; COLLA et al., 2008), são importantes pela possibilidade de substituição do uso do BHT (butilhidroxi tolueno), BHA (butilhidroxi anisol) e tBHQ (t-butil hidroquinona), antioxidantes sintéticos por antioxidantes naturais.

De acordo com Souza et al. (2006), Pilatty et al. (2007) e Canfield et al. (2008) em estudos da verificação da capacidade antioxidante da Spirulina platensis e da ficocianina em sistemas lipídicos, aquoso e "in vivo", essa microalga e o seu pigmento ficocianina vêm apresentando resultados que comprovam a atividade antioxidante, com atenuações significantes do potencial oxidativo, ou geração de radicais livres.

Os resultados da inibição da oxidação lipídica no armazenamento de pescado salgado vêm colaborar com estes estudos, favorecendo a possibilidade de substituição de antioxidantes sintéticos pelo uso de antioxidantes naturais, como a ficocianina. 


\section{CONCLUSÕES}

A ficocianina na concentração de $1,5 \%$ apresentou efeito antioxidante com relação ao índice de TBA estatisticamente iguais $(\mathrm{p}>0,05)$ ao antioxidante sintético BHT no armazenamento do pescado salgado, no tempo de $60 \mathrm{~d}$, com valores de 9,02 e 7,52 (mg kg-1), respectivamente.

A ficocianina pode atuar prevenindo ou minimizando a deterioração lipídica no processamento de pescado salgado, podendo ser uma alternativa para substituir o antioxidante sintético BHT.

\section{REFERÊNCIAS BIBLIOGRÁFICAS}

ABALDE, J. et al. Microalgas: cultivo y aplicaciones. La Coruña: Universidad de la Coruña, 1995.

ADEGOKE, G.O. et al. Antioxidants and lipid oxidation in food: a critical appraisal. Journal of Food Science and Technology, v.35, n.4, p.283-298, 1998.

ALENCAR, D.B; PIRES-CAVALCANTE, T. M. dos S.; SABOYA, J. P. de S.; SOUSA, M. B.; FARIAS, W. R. L.; SAKER-SAMPAIO, S. Teores de $\beta$-caroteno em suplementos e biomassa de Spirulina. Ciência e Agrotecnologia, v. 35, n. 2, p. 386-391, 2011.

ARAÚJO, J.M. Química de alimentos: teoria e prática.3.ed. Viçosa, MG: UFV, 2004. 458p.

ASGHAR, A. et al. Effect of salt on myoglobin derivatives in the sarcoplasmic extract from pre and post rigor beef in the presence of absence of mitochondria and microsomes. Meat Science, v.27, n.3, p.197-209, 1990.

ASHIE, I.N.A.; SMITH, J.P.; SIMPSON, B.K. Spoilage and shelf-life extension of fresh fish and shellfish.

Critical Reviews in Food Science and Nutrition, v.36, n.1/2, p.87-121, 1996.

BADR, H.M. Use of irradiation to control foodborne pathogens and extend the refrigerated market life of rabbit meat. Meat Science, v.67, n.4, p.541-548, Aug. 2004.

BERIAIN, M.J.; PEÑA, M.P.; BELLO, J. A study of the chemical components which characterize Spanish Saucisson. Food Chemistry, New York, v.48, n.1, p.31-37, 1993.
BRASIL. Ministério da Agricultura Pecuária e Abastecimento. Portaria no 52, de 29 de dezembro de 2000. Regulamento técnico de identidade e qualidade de peixe salgado e peixe salgado seco. Brasília, 2000.

CANFIELD, A.J. et al. Restrição calórica e a ficocianina no processo de envelhecimento de ratos sob parâmetros de extresse oxidativo. Revista Cientifica Jopef Online, v.1, n.3, maio 2008.

CHEN, Y.C. et al. Effects of dietary alpha-tocopheryl acetate on lipid oxidation and alpha-tocopherol content of novel omega-3-enhanced farmed rainbow trout (Oncorhynchus mykiss) fillets. LWT - Food Science and Technology, v.41, n.2, p.244-253, 2007.

COLLA, L.M.; BAISCH, A.L.M.; COSTA, J.A.V. Spirulina platensis effects on the levels of total cholesterol, HDL cholesterol and triglycerides in rabbits fed with with a hypercholesterolemic diet. Brazilian Archives of Biology and Technology, v.51, p.405-411, 2008.

COZZO, A.Z.; OETTERER, M.; GALLO, C.R. Effects of irradiation and refrigeration on the nutrients and shelflife of tilapia (Oreochromis niloticus). Journal of Aquatic Food Product Technology, Binghamton, v.12, n.3, p.85-102, 2003.

DURAN, R.M.; PADILLA, R.B. Actividad antioxidante de los compuestos fenólicos. Grasas e Aceites, v.44, n.2, p.101-106, 1993.

ESTRADA, J.E.P.; BESCÓS, P.B.; FRESNO, A.M.V. Antioxidant activity of different fractions of Spirulina platensis protein extract. Il Farmaco, v.56, n.5/7, p.497500, 2001.

FINKEL, T.; HOLBROOK, N. Oxidants, oxidative stress and the biology of ageing. Nature, v.408, p.239-247, 2000 .

GONZÁLEZ-FANDOS, E. et al. Microalga Spirulina: superalimento del futuro. Barcelona: Urano, 1994.

HASENHUETTL, G.L.; WAN, P.J. Temperature effects on the determination of oxidative stability with the metrohm rancimat. Journal of The American Oil Chemists. Society, v.69, n.6, p.525-527, June 1992. 
HENRIKSON, R. Microalga Spirulina: Superalimento del futuro. Ediciones S.A. Urano, Barcelona, Spain, 1994.

HOLMBERG, E.L. Viaje a missiones. Bol. Acad. Nac. Cienc., v. 10, pag. 222-387, 1887.

HUFFMAN, D.L.; LY, A.M.; CORDRAY, J.C. Effect of salt concentration on quality of restructured pork chops. Journal of Food Science, v.46, n.5, p.1563-1565, 1981.

INSTITUTO ADOLFO LUTZ. Normas analíticas do Instituto Adolfo Lutz: métodos químicos e físicos para análise de alimentos. 3.ed. São Paulo: O instituto, 1985. $533 p$.

ITÔ, Y.; SANCHES, L.; SILVA, D.R. Seasonal variation of the chemical composition of sardine. Contribuição Instituição Oceanográfica Universidade São Paulo-Tecnologia, São Paulo, n.6, p.1-8, 1969.

KELLEHER, S.D.; HULTIN, H.O.; WILHELM, K.A. Stability of mackerel surimi prepared under lipidstabilizing processing conditions. Journal of Food Science, v.59, n.2, p.269-271, 1994.

MELTON, S.L. Effects of feeds on flavor of red meat: a review. Journal of Animal Science, Champaign, v.68, p.4421-4435, 1990.

MORCUENDE, D. et al. Oxidative and lipolytic deteriotation of different muscles from free-range reared Iberian pigs under refrigerated storage. Meat Science, v.65, n.4, p.1157-1164, Dec. 2003.

MOREIRA, A.V. Influência dos compostos fenólicos de especiarias sobre a lipoperoxidação e o perfil lipídico de tecidos de ratos. Revista Nutrition, v.17, n.4, p.411-424, 2004.

NASSU, R.T. et al. Oxidative stability of fermented goat meat sausage with different levels of natural antioxidant. Meat Science, v.63, n.1, p.43-49, 2003.

OSAWA, C.C.; FELÍCIO, P.E.; GONÇALVES, L.G. Teste de TBA aplicado a carnes e derivados: métodos tradicionais, modificados e alternativos. Química Nova, São Paulo, v.28, n.4, p.655-663, 2005.
PEREIRA, A.V. et al. Estudo de estabilidade sob armazenamento da carne de ema (Rhea americana). Ciência e Tecnologia de Alimentos, Campinas, v.26, n.2, p.283-289, 2006.

PETENUCI, M. E. et al. Composição e estabilidade lipídica da farinha de espinhaço de tilapia. Ciência e Agrotecnologia, v. 34, n. 5, p. 1279-1284, 2010.

PILATTI, D. et al. Efeito da microalga Spirulina platensis (Arthrospira platensis) sobre a lipoperoxidação no hipocampo e perfil lipídico em ratos com hipercolesterolemia induzida. In: II Congresso nacional de Envelhecimento Humano, Passo Fundo. II Congresso nacional de Envelhecimento Humano. UPF, 2007

RAMALHO, V.C.; JORGE, N. Antioxidantes utilizados em óleos, gorduras e alimentos gordurosos. Química Nova, São Paulo, v.29, n.4, p.755-760, 2006.

RHEE, K.S.; SMITH, G.C.; TERRELL, R.N. Effect of reduction and replacement of sodium chloride on rancidity development in raw and cooked ground pork. Journal of Food Protection, n.46, p.578, 1983.

RICHMOND, A. Handbook of microalgal mass culture. 2.ed. Boston: CRC, 1990.

SALES, R.O.; MARTINS, C.B. Comparação entre dois métodos na determinação do índice de rancidez no pescado salgado-seco e defumado. Ciências Agronômicas, Fortaleza, v.19, n.1, p.161-165, 1988.

SANT'ANA, L.S.; MANCINI, F.J. Influence of the addition of antioxidants in vivo on the fatty acid composition of fish fillets. Food Chemistry, London, v.68, n.2, p.175-178, 2000.

SCHWARTZ, W.C.; MANDIGO, R.W. Effect of salt, sodium tripolyphophate and storage on restructured pork. Journal of Food Science, v.50, n.274, 1985.

SOUZA, F.T.; MARGARITES, A.C.; COLLA, L.M.; COSTA, J.A.V.; BERTOLIN, T. Avaliação do potencial antioxidante da ficocianina em sistema lipídico óleo de soja e azeite de oliva. Alimentos e Nutrição, v.17, n.3, p.275-279, jul./set. 2006. 
STATSOFT STATISTICA FOR WINDOWS. Computer programa manual. Versão 6.0. Tulsa, 2001.

TORRES, E.A.F.S. et al. Effect of salt on oxidative changes in pre-and-post-rigor ground beef. Meat Science, v.3, n.23, p.151, 1988.

TORRES, E.A.F.S. et al. Lipid oxidation in harqui: salted and dried beef. Food Chemistry, London, v.257, n.32, 1989.
UNIVERSIDADE FEDERAL DE SÃO PAULO. Escola Paulista de Medicina. Base de dados de nutrientes do USDA. Disponível em: $\langle\mathrm{http}: / / \mathrm{www}$ unifesp.br/dish servicos/nutri/nutri.php?id=2095;=4666e $=21601$. Acesso em: 23 nov. 2006 .

WICK, M.; MARRIOTT, N.G.; MCCLURE, K.E. Dietary supplementation of vitamin e affects the peroxide value of subcutaneous lamb fat. Journal of Muscle Foods, v.12, p.237-243, 2001. 